\title{
El quiasmo en el Praeceptum de san Agustín (IV)*
}

\author{
Pío DE Luis VizCAÍNO
}

SUMARIO: En el presente artículo, el autor presenta de forma sintética conclusiones que cabe extraer del análisis de los quiasmos detectados en el Praeceptum agustiniano.

ABSTRACT: In this article, the author presents in a synthetic way the conclusions that can be drawn from the analysis of the quiasmus found in the Praeceptum of Augustine.

Analizado ya el conjunto de quiasmos detectados en el Praeceptum agustiniano, llega el momento de hacer síntesis y extraer conclusiones. Varios son los aspectos que reclaman consideración.

\section{El dato numérico}

Sorprende la abundancia de quiasmos detectados en el código monástico agustiniano. Considerada su reducida extensión, los 24 (26) ejemplos de quiasmo parecen una cantidad elevada. Pero, además del simple dato numérico, es útil considerar otros. Uno, que el proceder de san Agustín no es idéntico en todos los capítulos del texto. Así, los capítulos primero, segundo, tercero y séptimo constituyen en su totalidad una estructura quiásmica; una estructura quiásmica se detecta también en los capítulos cuarto y sexto, pero de ella queda excluida la parte inicial del

* Para los tres artículos precedentes, consúltese Estudio Agustiniano 47 (2012) 427448; 48 (2013) 191-217; 49 (2014) 525-551. 
capítulo; el capítulo quinto carece de ese quiasmo globalizante, pero no faltan otros de menor extensión; el capítulo octavo es el único en el que no hemos advertido ninguno. Un segundo dato es que la existencia de un quiasmo que abarca la totalidad o casi del capítulo no impide la existencia en él de otros quiasmos, resultando una frecuente imbricación de los respectivos miembros.

\section{Las formas}

Los quiasmos contenidos en el Praeceptum, lejos de seguir un único modelo, ofrecen variedad de formas.

A) Si nos fijamos en la estructura, en el Praeceptum encontramos quiasmos de estructura concéntrica -sólo dos- y de estructura no concéntrica-los restantes-.

La estructura quiásmica habitual en el Praeceptum no es la que prefieren los autores bíblicos, en los que predomina la estructura concéntrica: los miembros exteriores apuntan a un miembro o miembros céntricos en el que o en los que el lector encuentra el núcleo de lo que el autor quiere trasmitir. En el código monástico agustiniano, sólo dos quiasmos tienen una estructura concéntrica. Como tales podemos considerar los quiasmos $\mathrm{n}^{\mathrm{o}} 6$ y 18 , ambos con cinco miembros. En realidad el quiasmo $\mathrm{n}^{\circ}$ 6 no encaja perfectamente en la categoría porque sus miembros exteriores son, sí, paralelos, pero no de paralelismo invertido; en vez del modelo ABCB'A' sigue el modelo ABCA'B'. Los demás quiasmos, de estructura no concéntrica, tienen sólo cuatro miembros (estructura $\mathrm{ABB}^{\prime} \mathrm{A}^{\prime}$ ), a excepción del $\mathrm{n}^{\circ} 14$ que consta de seis (estructura ABCC'B'A'). Cabe señalar todavía otras peculiaridades. La primera, propia del quiasmo $\mathrm{n}^{\circ}$ 1 , es el hecho de que cada uno de sus miembros es bimembre, si se acepta esta forma de hablar. La segunda es que los miembros exteriores de un mismo quiasmo constituyen en sí mismos otro quiasmo: es el caso del quiasmo $\mathrm{n}^{\mathrm{o}} 4$, constituido por los miembros exteriores del quiasmo $\mathrm{n}^{\mathrm{o}} 1$. Un caso parecido lo encontramos en el quiasmo $\mathrm{n}^{\circ} 17$. Sólo parecido porque en este último no sólo sus miembros exteriores, sino también sus miembros interiores constituyen otros quiasmos ( $\mathrm{n}^{\circ} 17 \mathrm{a}$ y $\left.17 \mathrm{~b}\right)$. Como característica se puede señalar también la frecuente desigualdad, por lo que a extensión se refiere, de sus miembros y la carencia habitual de simetría entre ellos. Otra peculiaridad del Praeceptum es incluir, dentro del texto que constituye la estructura quiásmica, otras estructuras paralelas, predominantemente antitéticas. De igual manera, a veces el quias- 
mo va precedido o seguido de una frase o simple sintagma, equivalente a la anacrusis en el ámbito musical ${ }^{1}$.

B) Si nos fijamos en los elementos que aparecen invertidos, estos pueden ser de naturaleza gramatical o de naturaleza conceptual.

Al hablar de naturaleza gramatical, nos referimos a las palabras en sus diversas morfologías y en sus distintas ordenaciones sintácticas. Al hablar de base conceptual nos referimos a cualquier concepto, ya sea considerado en abstracto, ya concretado en alguna realidad. Aplicada la distinción al Praeceptum, hay que decir que en él el paralelismo invertido tiene siempre una base conceptual, pero pocas veces sólo conceptual; que la base ordinaria es conceptual y gramatical a la vez, pero nunca sólo gramatical. Dicho en otros términos: lo que permite detectar el quiasmo es, en algunos casos, la inversión en paralelismo de determinados conceptos; de ordinario, ese paralelismo invertido de conceptos aparece también como inversión de formas gramaticales; pero ningún quiasmo se queda solo en pura floritura verbal sin un aporte de significado. En la elaboración del quiasmo tienen más peso los conceptos que su envoltura literaria. Sin que falten casos en que la inversión se lleve a cabo en parte con elementos gramaticales y en parte con elementos conceptuales.

\section{1) Componentes del quiasmo}

Estos son de diverso signo: oración a y b/oración c y d - oración d y c/oración b y a $\left(\mathrm{n}^{\mathrm{o}} 1\right)$; sintagma preposicional/verbo - verbo/sintagma preposicional $\left(\mathrm{n}^{\mathrm{o}} 2\right)$; oración de relativo+sintagma preposicional/ oración de relativo+adverbio - oración de relativo+adverbio/oración de relativo+sintagma preposicional ( $n^{\circ} 3$ ); oración/ sustantivos+adjetivos - adverbios [del mismo campo semántico que los sustantivos+adjetivos anteriores]/ oración ( $\left.n^{\circ} 4\right)$; verbo en plural/verbo en singular - verbo en singular/verbo en plural $\left(\mathrm{n}^{\mathrm{o}} 5\right)$; concepto a/verbo -concepto $\mathrm{b}$ - concepto a/ verbo $\left(\mathrm{n}^{\mathrm{o}} 6\right)$; concepto/adjetivo comparativo - adjetivo comparativo/concepto $\left(\mathrm{n}^{\mathrm{o}} 7\right)$; verbos/adjetivo - adjetivo/verbos ( $\left.\mathrm{n}^{\circ} 8\right)$; sustantivo+verbo en activa/verbo en pasiva - verbo en pasiva/verbo en activa+sustantivo ( $\left.n^{\circ} 9\right)$; sustantivo+adjetivo/sustantivo+adjetivo [pl.] - adjetivo+sustantivo/adjetivo+ sustantivo [sing.] ( $\left.\mathrm{n}^{\mathrm{o}} 11\right)$; verbos/verbo - verbo/verbos $\left(\mathrm{n}^{\mathrm{o}} 12\right)$; verbo/ verbo verbo/verbo ( $\left.\mathrm{n}^{\mathrm{o}} 13\right)$; sustantivo a/sustantivo b/sustantivo $\mathrm{c}$ - sustantivo $\mathrm{c} /$

\footnotetext{
${ }^{1}$ Pueden verse los quiasmos $n^{\mathrm{o}} 1,2,4,6,7,9,13,16,17,22,23$.
} 
sustantivo $\mathrm{b}$ /sustantivo a ( $\left.\mathrm{n}^{\mathrm{o}} 14\right)$; verbo/sustantivo - sustantivo/verbo $\left(\mathrm{n}^{\circ}\right.$ 15); adjetivo a /adjetivo $\mathrm{b}$ - adjetivo b/adjetivo a ( $\left.\mathrm{n}^{\mathrm{o}} 16\right)$; concepto/sustantivo - sustantivo/concepto ( $\left.\mathrm{n}^{\mathrm{o}} 17 \mathrm{a}\right)$; verbo a/verbo $\mathrm{b}$ - verbo c- verbo b/verbo a ( $\left.n^{\circ} 18\right)$; verbo/oración de relativo - oración de relativo/ verbo ( $\left.\mathrm{n}^{\mathrm{o}} 19\right)$; sustantivo a+verbo/sustantivo b+verbo - sustantivo b+ verbo/sustantivo a +verbo $\left(\mathrm{n}^{\mathrm{o}} 20\right)$; oración/sintagma preposicional - sintagma preposicional/oración ( $\mathrm{n}^{\mathrm{o}} 22$ ); sintagma preposicional/oración - oración/ sintagma preposicional $\left(\mathrm{n}^{\mathrm{o}} 23\right)$; verbo/concepto - concepto/verbo $\left(\mathrm{n}^{\circ} 24\right)$.

\section{2) Conceptos}

Como puede verse, los componentes que aparecen invertidos en los quiasmos del Praeceptum son variados. Y más si se tiene en cuenta que el afán de simplificación nos ha impedido señalar todos los particulares, por ejemplo recurriendo a la categoría de concepto cuando el autor se sirve de términos o expresiones sinónimas que son también categorías gramaticales. Pero en los quiasmos del Praeceptum no hay solo inversión de elementos gramaticales, sino también, y sobre todo, de conceptos. De hecho, aquellos pueden faltar, como hemos indicado, pero estos nunca.

Ante todo cabe señalar su gran variedad, indicadora de la riqueza de aspectos que el legislador considera implicados en la vida monástica. Primero señalamos aquellos pares de conceptos presentes en la primera secuencia que, al aparecer invertidos en la segunda, constituyen el quiasmo; en un segundo momento, añadimos otros pares de conceptos que, presentes en el texto del quiasmo, constituyen simples paralelismos; sin duda son de ayuda para conocer el pensamiento del autor del Praeceptum. Al precio de no multiplicar las divisiones, la clasificación que ofrecemos puede pecar de imprecisa en más de un caso.

a. Conceptos contrapuestos de forma especular, constitutivos del quiasmo, según ámbitos de pensamiento:

- Ámbito cosmológico: tiempo y espacio ( $\left.\mathrm{n}^{\mathrm{o}} 3\right)$; lugar y modo $\left(\mathrm{n}^{\mathrm{o}} 4\right)$.

- Ámbito antropológico: alimento físico y alimento espiritual $\left(\mathrm{n}^{\mathrm{o}} 6\right)$; sentimiento y razón $\left(\mathrm{n}^{\mathrm{o}} 7\right)$; interioridad y exterioridad $\left(\mathrm{n}^{\mathrm{o}} 11\right)$; lo común y lo personal ( $\left.\mathrm{n}^{\mathrm{o}} 16\right)$; decisión y consejo $\left(\mathrm{n}^{\mathrm{o}} 17\right)$; subjetividad y objetividad $\left(\mathrm{n}^{\mathrm{o}} 18\right)$; voluntad e inteligencia $\left(\mathrm{n}^{\mathrm{o}} 18\right)$; deseo y realidad $\left(\mathrm{n}^{\mathrm{o}} 8\right)$; conocimiento y ocultamiento $\left(\mathrm{n}^{\mathrm{o}} 12\right)$; salud espiritual y salud física $\left(\mathrm{n}^{\mathrm{o}} 17\right)$.

- Ámbito ético-moral: objetivo y medio ( $\mathrm{n}^{\mathrm{OS}} 1$ y 2 ); acción y pasión ( ${ }^{\circ}$ 9 ); acción y omisión ( $\left.\mathrm{n}^{\mathrm{o}} 13\right)$; lo conveniente y lo nocivo ( $\left.\mathrm{n}^{\mathrm{O}} 18\right)$; lo conve- 
niente y lo placentero ( $\left.\mathrm{n}^{\mathrm{o}} 19\right)$; objetivo y condición ( $\left.\mathrm{n}^{\mathrm{o}} 20\right)$; el mal/antídoto y su origen $\left(\mathrm{n}^{\circ} 22\right)$.

- Ámbito sociológico: grupo e individuo $\left(\mathrm{n}^{\mathrm{o}} 5\right)$; totalidad y parte $\left(\mathrm{n}^{\circ}\right.$ 8 ); autoridad jurídica y autoridad profesional $\left(\mathrm{n}^{\circ} 17\right)$; destinatario y acción $\left(\mathrm{n}^{\circ} 23\right)$; obediencia y ejercicio de la autoridad ( $\left.\mathrm{n}^{\circ} 24\right)$.

- Dualidad de opciones: sujeto a y sujeto b (n ${ }^{\mathrm{OS}} 3$ y 21$)$; cauce a y cauce b ( $\left.{ }^{\circ} 10\right)$; autoridad a y autoridad b (n 14$)$; acción a y acción b (nos 15 y 21); circunstancia a y circunstancia $b\left(n^{\circ} 21\right)$.

b. Conceptos contrapuestos en simple paralelismo en las secuencias:

- Ámbito metafísico/cosmológico: modelo y copia $\left(\mathrm{n}^{\circ} 1\right)$; realidad e ideal $\left(\mathrm{n}^{\circ} 2\right)$; diferencia e igualdad $\left(\mathrm{n}^{\circ} 3\right)$; partes y totalidad $\left(\mathrm{n}^{\circ} 6\right)$; tiempo y acción $\left(\mathrm{n}^{\mathrm{o}} 6\right)$; negación y afirmación $\left(\mathrm{n}^{\mathrm{o}} 7\right)$; plural y singular $\left(\mathrm{n}^{\mathrm{o}} 11\right)$; hecho y causa $\left(\mathrm{n}^{\mathrm{o}} 11\right)$; pasado y presente $\left(\mathrm{n}^{\mathrm{o}} 22\right)$.

- Ámbito antropológico: exterioridad e interioridad ( $\mathrm{n}^{\mathrm{os}} 3,5,23$ y 24); objetividad y subjetividad (nos 3, 7, 13 y 24).

- Ámbito ético-moral: medio y objetivo $\left(\mathrm{n}^{\circ} 2\right)$; obligatoriedad y libertad $\left(\mathrm{n}^{\circ} 5\right)$; lo agradable y lo eficaz ( $\mathrm{n}^{\mathrm{o}} 7$ ); sujeto y concupiscentia $\left(\mathrm{n}^{\circ}\right.$ 9); fuente del pecado y manifestación del pecado $\left(\mathrm{n}^{\mathrm{o}} 10\right)$; efecto y aspecto $\left(\mathrm{n}^{\mathrm{o}} 13\right)$; principio y aplicación ( $\mathrm{n}^{\mathrm{o}} 4$ y 15$)$; pecados (a y a') y criterio ( $\left.\mathrm{n}^{\mathrm{o}} 14\right)$; lo ideal y lo aconsejable ( $\left.\mathrm{n}^{\mathrm{o}} 15\right)$; lo positivo y lo negativo ( $\mathrm{n}^{\mathrm{o}} 16$ y 20); casuística/ razón y precepto $\left(\mathrm{n}^{\mathrm{o}} 18\right)$; lo relativo (no exigido) y lo absoluto (exigido) $\left(\mathrm{n}^{\mathrm{o}} 23\right)$.

- Ámbito religioso: sujeto a y sujeto $\mathrm{b}\left(\mathrm{n}^{\mathrm{o}} 12\right)$.

c. Conceptos contrapuestos de forma paralela en los dos miembros de una misma secuencia:

- Ámbito antropológico: objetividad y subjetividad ( $\left.\mathrm{n}^{\mathrm{o}} 22\right)$.

- Ámbito jurídico-moral: medio y objetivo $\left(\mathrm{n}^{\circ} 4\right)$; pecados a y pecados $\mathrm{b}\left(\mathrm{n}^{\mathrm{o}} 10\right)$; lo positivo y lo negativo $\left(\mathrm{n}^{\mathrm{o}} 13\right)$; objetivo y condición $\left(\mathrm{n}^{\mathrm{o}} 20\right)$; trasgresión y sanción $\left(\mathrm{n}^{\circ} 20\right)$.

- Ámbito sociológico: grupo a y grupo b ( $\left.\mathrm{n}^{\mathrm{o}} 6\right)$.

\section{d. Otros conceptos contrapuestos}

En el segundo miembro de la primera secuencia y el primer miembro de la segunda secuencia: interioridad y exterioridad $\left(n^{\circ} 2\right)$.

En el primer miembro de la primera secuencia y el segundo miembro de la segunda secuencia: subjetividad y objetividad $\left(\mathrm{n}^{\mathrm{o}} 12\right)$; ilusión truncada a e ilusión truncada $b\left(n^{\circ} 12\right)$.

C) Si nos fijamos en el significado, todos ofrecen alguna aportación al respecto; no se encuentra ninguno que sirva únicamente a la estética del texto. 
Una figura retórica -y el quiasmo es una entre otras muchas- es un recurso utilizado por el rhetor para llegar mejor al oyente o lector. Y, cuando el objetivo es enseñar, para hacerle llegar el mensaje que desea trasmitirle. Estudiar los quiasmos del Praeceptum es, por tanto, más que un simple ejercicio literario; significa abrir una vía que, junto a otras, facilite el acceso a una más plena interpretación de un texto ${ }^{2}$. La misma estructura quiásmica sirve retóricamente a ese fin.

Un recurso que facilita la retención en la mente es la asociación de conceptos opuestos, que a menudo son al actuar como dos despertadores a la vez, dado que uno llama de forma espontánea al otro. Ahora bien, por definición, es propiedad inherente al quiasmo el yuxtaponer conceptos o correlativos contrapuestos. Por otra parte, el quiasmo facilita la ubicación de los conceptos en lugares estratégicos suscitando la atención y facilitando la retención. La fuerza del quiasmo radica precisamente en la posibilidad que da al autor para ubicar sus conceptos en los lugares que la psicología considera estratégicos. Por su función estructurante, el quiasmo tiene aptitudes expresivas que le son propias. Es sabido que la capacidad retentiva de la memoria guarda relación con la atención de la mente. Con el fin de despertar esa atención, el rhetor tiene en cuenta constataciones de índole psicológica. Si lo primero con que entramos en contacto nos impacta, es probable que ello reclame nuestra atención, quedando particularmente grabado en la mente; de igual manera, si lo último que percibimos es más impactante que lo anterior, lo más probable es que sea eso lo que mejor retenga nuestra memoria o, quizá, lo único ${ }^{3}$.

Dejamos ya el planteamiento teórico para pasar a presentar el modo de actuar de san Agustín al respecto. Por su importancia semántica, destacamos tres tipos de relaciones internas: a) la relación entre los miembros

${ }^{2}$ Según J. Breck, advertir el quiasmo representa ya un primer nivel de interpretación (Chiasmus as a Key of Biblical Interpretation, p. 267).

3 Ambas constataciones están recogidas, respectivamente, en estos dos refranes españoles "el que da el primero da dos veces" y "el que ríe el último ríe dos veces". El primero de ellos puede ponerse en relación con el primer miembro exterior del quiasmo, y el segundo, con el segundo miembro exterior. Los miembros exteriores representan, a la vez, lo primero y lo último con lo que se entra en contacto; lo que, por consiguiente, es probable que quede más grabado en la mente. Pero cuando, dentro de una identidad básica, hay variantes entre los dos términos exteriores, el refrán aplicable es el segundo: la idea que vale, aquella con la que hay que quedarse, es la última; la primera ha servido sólo de introducción necesaria. 
exteriores y los interiores; b) la relación entre los dos miembros exteriores; c) la relación entre la primera y la segunda secuencia.

\section{1) Relación semántica entre los miembros exteriores y los interiores}

Desde el punto de vista semántico, el legislador otorga más entidad a los miembros exteriores que a los interiores. Es característica bien perceptible en el Praeceptum. Es a los primeros a los que hay que dirigir la mirada para conocer lo que domina la mente del legislador. En ellos ubica aquello a lo que asigna más importancia y lo que desea inculcar a los monjes, ya sea en positivo, tratando de promoverlo, ya sea en negativo, tratando de desterrarlo. Los miembros interiores los reserva generalmente para señalar diversas circunstancias relacionadas con el objetivo, positivo o negativo, que pretende alcanzar. Prueba de lo afirmado son los abundantes ejemplos que ponemos a continuación, en los que el primer dato se encuentra en los miembros exteriores y el segundo ${ }^{4}$ en los interiores:

- Los miembros exteriores señalan los objetivos -mayor entidad-, y los miembros interiores, los medios -menor entidad-: unidad de almas y corazones (casa/templo) - tener todo en común y distribución según necesidad ( $\left.{ }^{\circ} 1\right)$; in unum/in Deum - habitar juntos/habitar en unidad de alma y corazones $\left(n^{\circ} 2\right)$.

- Los miembros exteriores contemplan la categoría tiempo -más significativa en un marco histórico-salvífico-; los interiores, la categoría espacio -menos significativa- $\left(\mathrm{n}^{\mathrm{o}} 3\right)$.

- Los miembros exteriores señalan la acción -psicológicamente más valorada- y los miembros interiores, la pasión -menos valorada-: ver/desear mujeres - ser deseado por ellas ( $\left.n^{\circ} 9\right)$; corregir - perecer (aquí con distintos sujetos) ( $\left.\mathrm{n}^{\mathrm{o}} 13\right)$.

- Los miembros exteriores señalan la interioridad -el valor- y los miembros interiores, la exterioridad -el contravalor-: alma/corazón puro ojo impuro $\left(n^{\circ} 11\right)$.

- Los miembros exteriores señalan el descubrimiento -formulación en positivo- y los miembros interiores, la no ocultación -formulación en negativo- la mirada lasciva es descubierta - no queda oculta ( $\left.n^{\circ} 12\right)$.

- Los miembros exteriores señalan la acción -lo positivo- y los miembros interiores, la inacción -lo negativo-: delatar la falta - silenciarla $\left(\mathrm{n}^{\mathrm{o}}\right.$ 13).

\footnotetext{
${ }^{4}$ Nos referimos a los datos separados por guión (-), no por barra (/).
} 
- Los miembros exteriores señalan lo común -el valor- y los miembros interiores, lo particular -el contravalor-: preferencia a los bienes comunes - preferencia a los bienes particulares $\left(n^{\circ} 16\right)$.

- Los miembros exteriores señalan la decisión -más eficiente- y los miembros interiores, el consejo -menos eficiente-: la decisión del prepósito - el consejo del médico ( $\left.\mathrm{n}^{\circ} 17\right)$.

- Los miembros exteriores señalan lo conveniente -el valor- y los miembros interiores, lo placentero -aquí el contravalor-: la conveniencia de ir a los baños - el solo placer de ir a ellos ( $\left.n^{\circ} 19\right)$.

- Los miembros exteriores señalan el objetivo -mayor entidad- y los miembros interiores, la condición -menor entidad-: recibir los códices respetar el horario $\left(\mathrm{n}^{\mathrm{o}} 20\right)$.

- Los miembros exteriores señalan el tiempo del perdón -más importante-, los miembros interiores, el modo -menos importante- $\left(\mathrm{n}^{\mathrm{o}} 21\right)^{5}$.

- Los miembros exteriores señalan la obediencia -el objetivo- y los miembros interiores, la autoridad -el medio-: ejercicio de la obediencia ejercicio de la autoridad $\left(n^{\circ} 24\right)^{6}$.

Asimismo, cuando se trata de dos acciones concretas, los miembros exteriores señalan la acción más valorada por el legislador, y los miembros interiores, la menos valorada por él.

- Los miembros exteriores se refieren a la oración en común; los interiores, a la oración individual $\left(\mathrm{n}^{\mathrm{o}} 5\right)^{7}$.

- Los miembros exteriores ordenan poner la ropa a disposición de todos; los miembros interiores, su cuidado común ( $\left.{ }^{\circ} 15\right)^{8}$.

- Los miembros exteriores miran por la salud moral; los interiores, por la salud física $\left(\mathrm{n}^{\mathrm{o}} 17\right)^{9}$.

5 Tras haber afirmado que la ira puede degenerar en odio, al legislador urge ante todo la inmediatez del perdón.

${ }^{6}$ Hay autores que consideran propio de san Agustín haber dado más relieve al ejercicio de la autoridad que al de la obediencia. Si nos atenemos al criterio señalado, la verdad sería lo contrario.

7 Para el legislador, la oración común es prioritaria respecto a la oración privada (en el oratorio), que sin duda promueve, pero que supedita a ciertas condiciones.

8 El ponerla a disposición de todos indica un nivel mayor de compromiso con la comunidad que el simple aceptar que el cuidado de la misma sea común.

${ }^{9} \mathrm{El}$ interés principal del prepósito es la salud moral-espiritual de los monjes, mientras que el médico sólo se ocupa de la salud física. 
- Los miembros externos ordenan pedir perdón; los interiores, perdonar $\left(\mathrm{n}^{\mathrm{o}} 21\right)^{10}$.

- Los miembros exteriores se centran en la entrega de los códices, los interiores, en el respeto del horario $\left(\mathrm{n}^{\circ} 20\right)^{11}$.

Lo mismo sucede cuando se trata de dos sujetos; en este caso los miembros exteriores señalan el más valorado por el legislador, y los miembros interiores, el menos valorado por él:

- Los miembros exteriores se ocupan del monje "rico" -más valorado-, y los interiores, del monje pobre-menos valorado- $\left(\mathrm{n}^{\mathrm{o}} 3\right)^{12}$.

- Los miembros exteriores se fijan en el monje (sus sentimientos); los miembros interiores en el legislador (sus razones) $\left(\mathrm{n}^{\circ} 7\right)^{13}$.

- Los miembros exteriores aluden a los sujetos; los interiores, a su número $\left(\mathrm{n}^{\mathrm{o}} 8\right)^{14}$.

- Los miembros exteriores señalan la autoridad propia del monasterio: el prepósito; los miembros interiores, una autoridad adjunta: el presbítero ( $\left.\mathrm{n}^{\mathrm{o}} 14\right)$; o, respectivamente, a la autoridad propia: el prepósito, y al consejero en asuntos de salud: el médico ( $\left.\mathrm{n}^{\circ} 17\right)$; o a los monjes, y a la autoridad $\left(\mathrm{n}^{\circ} .24\right){ }^{15}$.

- Los miembros exteriores se refieren al ofensor -más problemático-, y los interiores, al ofendido -menos problemático- $\left(\mathrm{n}^{\mathrm{o}} 21\right)^{16}$

10 En este caso, no se trata de que el pedir perdón sea más valorado y el concederlo, menos, sino de que el legislador, por las razones que sean, insiste más en la dificultad y necesidad de pedir perdón que en la de concederlo, de lo que es prueba también la extensión dedicada a una y otra acción.

11 Está claro que lo importante es la entrega y recepción de los códices; respetar el horario es sólo una condición.

12 El legislador puede haberse atenido a la valoración social, pero también a una valoración religioso-monástica. Al santo el rico le ofrece más garantías vocacionales que el pobre. La razón: al primero el ingreso en el monasterio le significó renunciar a algo; no así al segundo que al que, posiblemente, le significó adquirir algo que no poseía (cf. op. mon. $22,25 ; 25,33)$.

13 Lo importante son los monjes; el legislador aparece en función de ellos.

14 Obviamente, lo valioso son las personas, independientemente de su número.

15 En el primer caso, el legislador pone delante al prepósito, en cuanto autoridad que rige el monasterio, mientras que el presbítero solo ha de intervenir en casos específicos (cf. Praec. $\mathrm{n}^{\mathrm{o}} 45$ ); en el segundo caso antepone de nuevo al prepósito, en cuanto autoridad que rige el monasterio, mientras que el médico es un simple consejero. En el tercer caso, en cambio, son los monjes quienes son antepuestos al prepósito, la autoridad del monasterio, porque esta existe en función de aquellos y no al revés.

16 Véase lo dicho antes (nota 10) a propósito del pedir y conceder el perdón. 
- Los miembros exteriores se centran en el destinatario: monje/Dios; los interiores, en la acción: pedir perdón (nº 23) ${ }^{17}$.

\section{2) Relación semántica entre el primer miembro y el segundo miembro exteriores}

Hemos visto el mayor valor que en los quiasmos del Praeceptum representan los miembros exteriores respecto de los interiores. Un dato que ha de ser completado con este otro: comparados los dos miembros externos, el segundo es privilegiado respecto al primero. Aquel suele representar un peldaño más en la escala del valor propuesto en el primero. En los ejemplos que siguen a continuación los ordinales "primero" y "segundo" se refieren siempre a los miembros exteriores del quiasmo.

- El primero habla de casa, el segundo, ya de templo (no 1 y 4) 18 .

- El primero habla de in unum, el segundo de in Deum ( $\left.\mathrm{n}^{\mathrm{o}} 2\right)^{19}$.

- El primero habla de congregari, el segundo de honorare $\left(\mathrm{n}^{\circ} 4\right)^{20}$.

- El primero expresa un sentimiento negativo; el segundo, uno positivo $\left(\mathrm{n}^{\circ} 7\right)^{21}$.

- El primero expresa el deseo; el segundo, la realidad $\left(\mathrm{n}^{\circ} 8\right)^{22}$.

- El primero emplea el término animus, el segundo cor $\left(\mathrm{n}^{\circ} 11\right) 23$.

- El primero se refiere al ver del hombre; el segundo, al ver de Dios $\left(n^{\circ} 12\right)^{24}$.

- El primero indica quién aporta el criterio; el segundo, quién toma la decisión (nº 17) 25.

$17 \mathrm{Al}$ aparecer Dios por medio, es lógico que obtenga el lugar preferente y, con él, el hombre, receptor, como él, de la acción.

18 El legislador pasa de la casa como edificio material o como realidad social a la casa de Dios, el templo, que son los monjes mismos.

19 Según el legislador, los monjes pasan de tener en su mente la unidad dentro del grupo humano a tener a Dios, fundamento de una unidad superior.

20 Según el legislador los monjes han de pasar de un simple estar reunidos físicamente a un estar juntos que implica honrar a Dios los unos en los otros.

${ }^{21}$ El legislador propone a los monjes pasar de la sensación de ser víctimas de un atropello y una injusticia a ver en la misma realidad un motivo del que congratularse.

$22 \mathrm{El}$ legislador pasa del deseo de unos monjes a la realidad que viven otros.

23 El legislador pasa de un término de la tradición filosófica -el primero- a uno de la tradición bíblica -el segundo-.

${ }^{24}$ El legislador pasa del hombre a Dios: aquel no ve la falta ajena, a este no se le oculta.

25 El legislador contempla al prepósito en dos momentos: primero, como capacitado para decidir -suyo es el criterio-; luego, habiendo tomado ya la decisión. 

18) 26 .

- El primero se refiere a la voluntad; el segundo, a la inteligencia $\left(\mathrm{n}^{\circ}\right.$

- El primero habla de palabras; el segundo, de medicamentos $\left(\mathrm{n}^{\circ} 22\right){ }^{27}$.

- El primero se refiere al mal; el segundo, al antídoto $\left(\mathrm{n}^{\mathrm{o}} 22\right)^{28}$.

- El primero se refiere al hombre; el segundo, al Señor $\left(\mathrm{n}^{\mathrm{o}} 23\right)^{29}$.

3) Relación semántica entre la primera y la segunda secuencia de los quiasmos

Entre la primera secuencia y la segunda hay una relación, que no siempre se manifiesta de la misma manera. La relación es de dependencia de la segunda respecto de la primera. Pero esa dependencia responde a las diferentes "lógicas" que a continuación señalamos.

- La que va del modelo a la copia $\left(\mathrm{n}^{\mathrm{o}} 1\right)$.

- La que va del hecho a la finalidad del mismo $\left(\mathrm{n}^{\mathrm{o}} 2\right)$.

- La que pasa de una realidad objetiva/exterior a una realidad subjetiva/interior $\left(\mathrm{n}^{\circ} 3,13,24\right)$.

- La que pasa de lo que diferencia a lo que unifica $\left(n^{\circ} 3\right)$.

- La que va del principio a su aplicación ( $\mathrm{n}^{\circ} 4$ y 15).

- La que va de lo general a lo concreto $\left(n^{\circ} 5\right)$.

- La que va de la causa al efecto o del efecto a la causa ( ${ }^{\circ} 11$ y 18).

- La que pasa de lo referente al hombre a lo referente a Dios $\left(n^{\circ} 12\right.$, 23).

- La que pasa de lo menos grave a lo más grave $\left(\mathrm{n}^{\circ} 14\right)$.

- La que va de lo ideal a lo factible ( $\left.\mathrm{n}^{\circ} 15\right)$.

- La que pasa de la prescripción a la prohibición ( $\mathrm{n}^{\mathrm{o}} 16$ y 20).

- La que pasa de lo pretérito a lo presente $\left(\mathrm{n}^{\circ} 22\right)$

- La que pasa de la libertad a la obligación ( $\left.{ }^{\circ} 23\right)$.

Gramaticalmente la relación de la segunda secuencia con la primera se expresa a veces con conjunciones de subordinación: final $\left(u t: \mathrm{n}^{\circ} 1\right)$; causal (quia: $\left.\mathrm{n}^{\circ} 11\right)$; condicional (si: $\left.\mathrm{n}^{\circ} 13\right)$, o de coordinación: adversativa

26 El legislador contempla primero lo que quiere el monje y luego lo que él -erróneamente (creditur)- entiende, razón de que quiera lo que quiere. La voluntad sigue a la inteligencia.

27 El legislador transforma las palabras (uerba emittere) en medicamentos (medicamenta proferre).

28 El legislador pasa del mal, las palabras ofensivas, a lo que lo cura, palabras que solicitan el perdón.

29 El legislador pasa del pedir perdón al hombre a pedírselo al Señor. 
(tamen: $\mathrm{n}^{\mathrm{o}} 14 ;$ sed: $\mathrm{n}^{\mathrm{o}} 23$; uero: $\left.\mathrm{n}^{\circ} 24\right)$. O también con un pronombre interrogativo (quid: $\mathrm{n}^{\mathrm{o}} 12$ ).

Diverso es el caso en los pocos quiasmos de estructura concéntrica. En ellos, la mirada no ha de dirigirse a los miembros externos, sino al miembro central para descubrir el valor que más potencia el legislador o el vicio que desea desterrar.

- La recomendación del alimento espiritual -la escucha de la palabra de Dios- va precedida y seguida de las prescripciones referentes al alimento físico $\left(\mathrm{n}^{\mathrm{o}} 6\right)$.

- La recomendación de no obedecer a la cupiditas va precedida en los miembros interiores de la referencia a lo que se cree que conviene (expedit) y seguida de la referencia a lo que objetivamente daña (noceat) y en los miembros exteriores de la referencia a la voluntad (uelit) y a la inteligencia (creditur) $\left(\mathrm{n}^{\mathrm{o}} 18\right)$.

\section{Función del quiasmo}

El recurso al quiasmo en un texto puede obedecer a razones estructurales o estéticas, o a ambas a la vez. Efectivamente, no cabe dudar de las posibilidades que brinda el quiasmo para estructurar un texto. En sí mismo es ya una estructura literaria. De hecho, por simple variatio, hemos venido sustituyendo el sustantivo "quiasmo" por el sintagma "estructura quiásmica". Pero una estructura con unas características específicas en cuanto al orden que se ha de seguir en la exposición de las ideas o de las palabras mismas. Justamente por esto, aporta siempre un "tinte retórico" ${ }^{30}$. Tampoco cabe duda de que el quiasmo contribuye al ornato del discurso hablado o escrito. Esa contribución puede consistir en introducir la variedad que evita la monotonía, en proporcionar la brevedad que sortea el hastío, en favorecer la contraposición de dos o más puntos de vista. Por otra parte, el quiasmo facilita el yuxtaponer términos en contraste o el asociar términos correlativos. El quiasmo se constituye en medio de transmisión de ideas alternativo a la palabra. Lo que en el discurso hablado puede ser expresado mediante el lenguaje del cuerpo, un ademán, un gesto o el tono de la voz, en un texto escrito puede hallar expresión en el

30 R. B. Steele. Dato tomado de J. W. Welch, Chiasmus in Ancient Greek and Latin Literatures, p. 259. 
quiasmo; dígase lo mismo de los silencios o énfasis. Aunque sirviéndose de palabras, cabe entenderlo como un lenguaje metaverbal.

Pero volvamos al texto agustiniano. La función que en él tiene el quiasmo es esencialmente estructurante, no estética, aunque no falten ejemplos que encajan también dentro de esta categoría. Como ya indicamos, estructura determinados capítulos del Praeceptum en su totalidad, en buena parte otros, además de unidades de texto menores dentro de los diversos capítulos. El quiasmo permite al legislador exponer sus ideas en determinado orden, elegido por él. Eso es lo que le importa. Asegurado ese orden de ideas por medio del quiasmo, se desinteresa de otros aspectos que podrían considerarse como estéticos, tales como la desproporción y falta de simetría entre los diversos miembros. De hecho, de ninguno de los quiasmos del texto agustiniano se puede afirmar que obedezca exclusivamente al ornato del texto. Tal intención en el autor exigiría una forma más inmediatamente perceptible, aunque no deje de tener su encanto descubrir una figura retórica encubierta. No obstante su función estructurante, el quiasmo agustiniano no se identifica con el hysteron proteron; es más que simple orden de exposición temática; el quiasmo es ámbito literario dentro del cual se desarrolla el pensamiento agustiniano.

El quiasmo permite al legislador focalizar el núcleo de su pensamiento $\mathrm{y}$, al mismo tiempo, dotar de dinamismo a sus propuestas. La figura retórica aparece así como el recurso literario elegido por el legislador para mostrar diversas etapas en el caminar espiritual de los monjes.

\section{IV. ¿Espontaneidad o artificio?}

John W. Welch considera el quiasmo como una de las estructuras profundas de que hablan los estructuralistas ${ }^{31}$. Nosotros, pensando sólo en san Agustín y juzgando únicamente por el Praeceptum, nos limitamos a sostener que el quiasmo es una estructura literaria bien asentada en su mente. La pregunta de si el recurso tan frecuente al quiasmo es fruto de la espontaneidad o resultado de un artificio literario no creemos que halle

${ }^{31}$ Chiasmus in Ancient Greek and Latin Literatures, en JoHN. W. WelCH (ED.), Chiasmus in Antiquity. Structures, Analyses, Exegesis, Research Press Reprint Edition, Provo, UTA 1998, 250-268: 255. Hablando más en concreto del paralelismo concéntrico, J. Breck lo considera una "estructura profunda de la mente humana" (Chiasmus as a Key of Biblical Interpretation, en SVTQ 43 [1999] 249-267:267). 
la respuesta adecuada en clave disyuntiva. $\mathrm{Si}$ aplicamos el criterio propuesto por J. W. Welch -"cuando un pensamiento o una corta sentencia aparece plenamente encerrada en un quiasmo, la figura se considera natural y no afectada" 32 - los quiasmos agustinianos del Praeceptum, caen en su gran parte en la categoría de artificiales. El artificio lo prueban también algunas constantes detectadas en relación con sus contenidos doctrinales, ya indicadas. Tal constancia permite suponer cierto nivel de elaboración consciente. Pero, contra el criterio de J. Welch, podemos considerar como prueba de la falta de artificio la frecuencia y la desigualdad de los miembros, así como la falta de simetría. El proceder de san Agustín podemos resumirlo en estos términos: el artificio se manifiesta principalmente en el ámbito conceptual, no en el formal.

\section{Conclusión}

Ponemos término al estudio de los quiasmos en el Praeceptum agustiniano con dos conclusiones que son como dos caras de una misma moneda. Una formulada en relación al texto; otra, en relación al autor.

Un texto, aunque no aparezca como especialmente cualificado, cuando su autor lo es, siempre oculta más riqueza de la que manifiesta. Salvadas las diferencias, se puede aventurar un juicio semejante al que hace Agustín sobre la Escritura: "de entrada humilde, de interior sublime" (Conf. 3,5,9). No nos referimos, por supuesto, ahora al ámbito semántico, sino al literario. La retórica había alimentado a Agustín; primero su espíritu en la época de estudiante y luego incluso su cuerpo porque, como profesor, vivía de ella. Y la retórica impregnó siempre y todo cuanto salía de sus manos, si no de una forma, de otra; si no siempre impresiona al que solo mira, sí sorprende al que también busca. Tal ha sido el caso del Praeceptum agustiniano en relación con el quiasmo. Sin olvidar nunca que, en san Agustín, la retórica nunca es pura exhibición de habilidades específicas; es también servicio a la verdad.

Un autor, y más si es especialmente cualificado, sabe siempre encontrar nuevos medios para expresar su pensamiento. El escritor no cuenta, como el orador, con el poder de comunicación de los ademanes, gestos, y tono de voz, pero su arte le permite aprovechar otros recursos. Así, mediante una estratégica ubicación en la frase o una calculada asociación

32 Cf. Chiasmus, p, 159. 
entre ellas puede hacer que las ideas ganen o pierdan rango en la percepción del lector. Las palabras mismas pueden adquirir un plus de significado, siempre puntual, sin necesidad de que cambie su semántica. Por significado no se entiende solo lo que comunican, sino también la fuerza con que lo comunican. Todo es realidad a la que sirve el quiasmo. Y es lo que hemos querido mostrar en las páginas que preceden. 